\title{
AN ELECTRONIC PHONOCARDIOGRAPH EMPLOYING A DOUBLE-BEAM CATHODE-RAY OSCILLOGRAPH AS THE RECORDING DEVICE
}

\author{
BY \\ F. W. CAMPBELL, A. W. SLOAN, AND A. M. ANDREW \\ From the Institute of Physiology, University of Glasgow \\ Received September 17, 1951
}

\begin{abstract}
A phonocardiograph employing a cathode-ray tube is the theoretical ideal since the natural frequency of the instrument is sufficiently high to avoid distortion of the vibrations to be recorded, the amplitude of the deflections is directly proportional to the movements which excite them, and the cathode-ray beam has minimal inertia and is fully damped.

With any of the popular recording devices it is possible to obtain some sort of record of the vibrations produced in the front of the chest by the beat of the heart. Such a. record has little resemblance to the sounds heard on cardiac auscultation unless the low-frequency elements are filtered off so that the remaining deflections on the record correspond to vibrations of audio-frequency. A faithful record of vibrations of all frequencies is called a linear phonocardiogram, one in which the low frequency vibrations are attenuated as by the introduction of an ordinary stethoscope is called a stethoscopic phonocardiogram, and a record in which the low-frequency attenuation of the stethoscope as well as the poor low-frequency response of the human auditory mechanism have been imitated is called a logarithmic phonocardiogram. Such logarithmic attenuation of the low-frequency vibrations in imitation of that inherent in auscultation is most conveniently achieved by electrical filters.

An electronic phonocardiograph using a single-beam cathode-ray oscillograph was employed by Boone (1940). This instrument merely recorded the linear phonocardiogram without any reference tracing. Kountz et al. (1940) recorded linear phonocardiograms with a cathode-ray oscillograph; the electrocardiogram (lead II) was recorded as a reference tracing at the same time. Donovan (1944) employed a double-beam cathode-ray oscillograph as the recording device. Electrical filters were incorporated in the amplifier system to give sufficient attenuation of the low frequencies for the recording of stethoscopic phonocardiograms, and the reference tracing recorded on the other beam was either the electrocardiogram or the jugular venous pulse. Later, in 1948, Donovan preferred the logarithmic phonocardiogram because it is the only one comparable to auditory findings, and the phlebogram was preferred as the reference tracing because the electrical activity of the heart as indicated by the electrocardiogram does not bear a constant time-relationship to mechanical events such as heart sounds and pulse waves.
\end{abstract}

\section{Description of Present Instrument}

Fig. 1 is a block diagram of the present instrument. There are four main units, the preamplifier, the audiofrequency amplifier, the low-frequency amplifier, and the double-beam cathode-ray oscillograph. These will now be described in turn.

Preamplifier. An amplifier giving at will any one of a range of suitable frequency response curves has been described by Cowen and Parnum (1949). As their circuit was designed to activate a string galvanometer, we had to modify the circuit for use with the cathode-ray oscillograph (Fig. 2). 


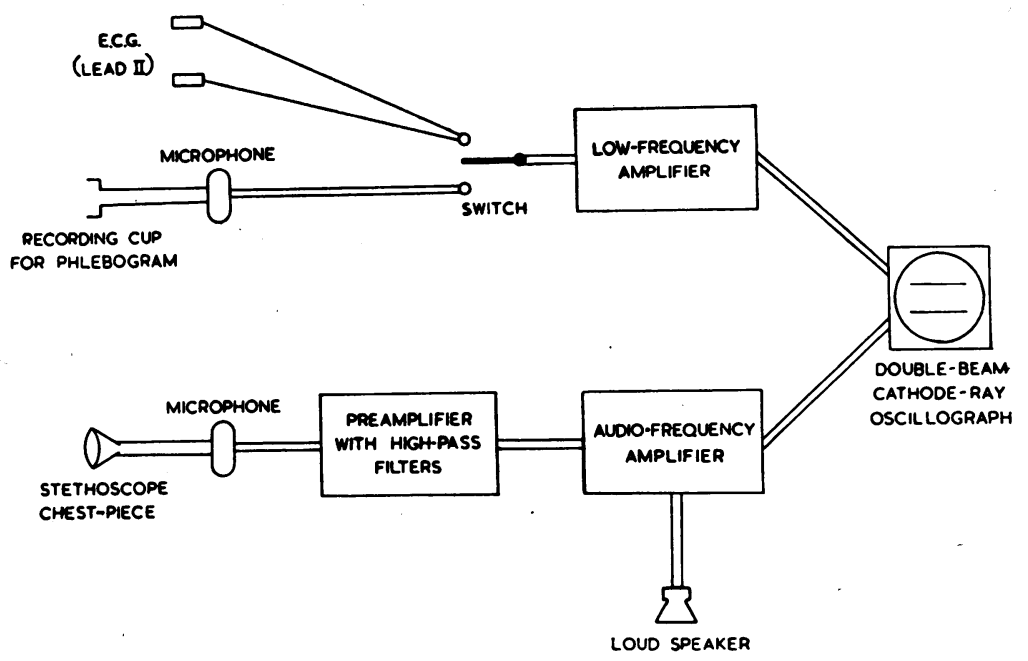

Fig. 1.-Block diagram of electronic phonocardiograph.

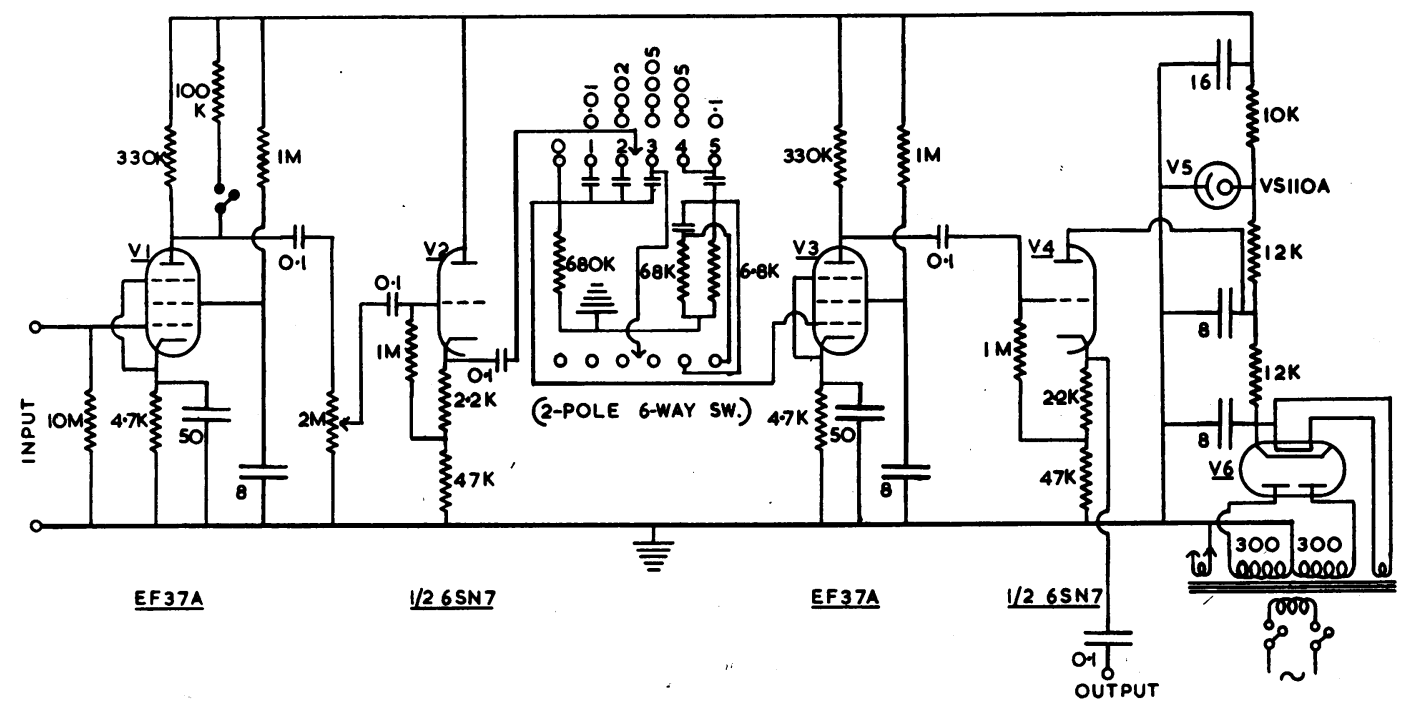

Fig. 2.-Circuit diagram of preamplifier. Capacitor values in microfarads. Resistor values in $\mathbf{M}\left(10^{6} \mathrm{ohms}\right)$ and $\mathrm{K}\left(10^{3} \mathrm{ohms}\right)$.

The output from a crystal microphone is applied to the grid of V1, which is connected as a conventional stage of amplification. The switch in the anode circuit gives a step control of gain (ratio of 4 to 1) by reducing the anode load, and the 2-megohm potentiometer gives continuous gain control. $\mathrm{V} 2$ is a cathode follower whose low output impedance facilitates the design of the filter network.

At setting 0, a linear phonocardiogram is obtained. At settings 1,2 , and 3, single-stage resistance-capacitance filters, having time-constants of $0.0068,0.0013$, and 0.00034 seconds respectively, are provided. At settings 4 and 5 a two-stage and three-stage filter respectively are introduced, the time-constant of each additional section being 0.00034 seconds. These filter values closely follow those of Cowen and Parnum. 
The filter network is followed by a further stage of amplification (V3) and another cathode follower (V4) so that the output signal is unaffected by the capacitance of the screened lead to the final amplifier.

The power supply is conventional. V5 stabilizes the H.T. voltage supplied to V1, V2 and V3. The supply to V4 must be taken from another point in the smoothing circuit to decouple this stage from the earlier ones and prevent oscillation.

Frequency response curves for the preamplifier are shown in Fig. 3, relative to the response at 1000 cycles per sec. (c/s). The broken line shows the response of the human ear, relative to its response at $125 \mathrm{c} / \mathrm{s}$ based on a curve given by Fletcher (1948).

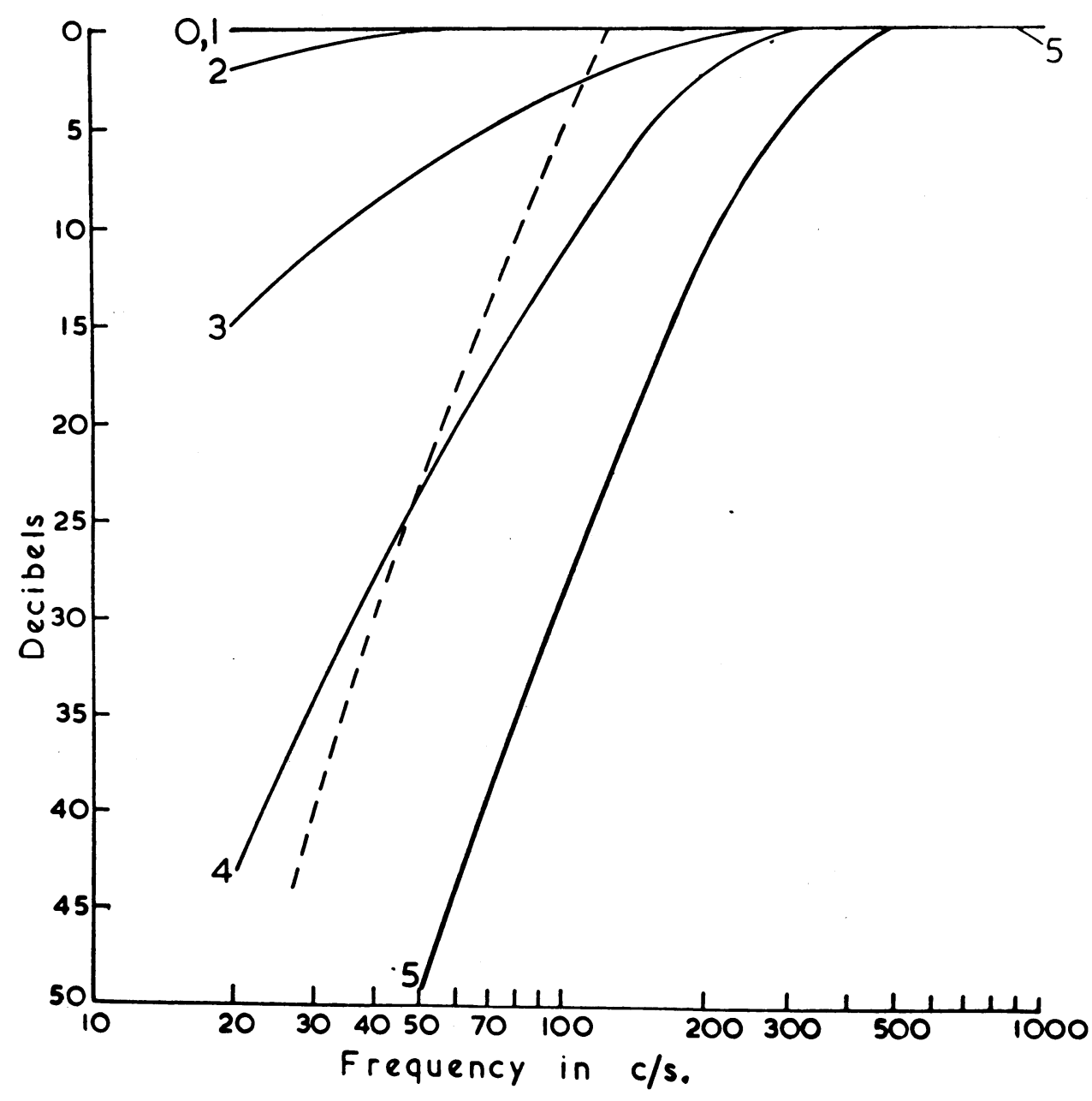

FIG. 3.-Frequency response curves of preamplifier compared with normal auditory response. The figures $0,1,2,3,4$, and 5 refer to positions of filter switch on preamplifier. Interrupted line represents normal auditory response (Fletcher, 1948).

For any filter setting, the maximum gain of the amplifier is approximately 72 decibels at $1000 \mathrm{c} / \mathrm{s}$. With the filter set to position 4, and gain set at maximum, the maximum output voltage from the heart sounds of a typical subject is about $0.45 \mathrm{v}$. peak-to-peak, the lowest encountered being about $0 \cdot 3 \mathrm{v}$. 
Audio-frequency Amplifier. The output of the preamplifier can be applied to the amplifiers of a commercial cathode-ray oscillograph. In our apparatus, since an output suitable to drive a loud speaker is desired, the preamplifier output is connected to a standard audio amplifier, namely that described by Williamson (1951). The output transformer has been wound with a 600 -ohm secondary winding in addition to the low impedance winding for the speaker, enabling both the cathode-ray tube and the speaker to be supplied by the same amplifier.

Low-frequency Amplifier. This is a fairly straightforward biological amplifier which has given excellent results in the recording both of the cardiogram and of the phlebogram. It consists of a stage of push-pull amplification using a 6SN7 double triode, followed by a Toennies compressor stage, also using a 6SN7, and followed by two stages of single-sided amplification using SP61 pentodes. The amplifier is resistance-capacitance coupled throughout, with time constants of $4 \mathrm{sec}$. A stepped gain control is provided between the second and third stages, and variable negative feed-back over the two final stages provides a continuous control of gain. The first two stages are battery operated both for L.T. and H.T. supplies. The two later stages are mains driven, with A.C. heater supply and stabilized H.T.

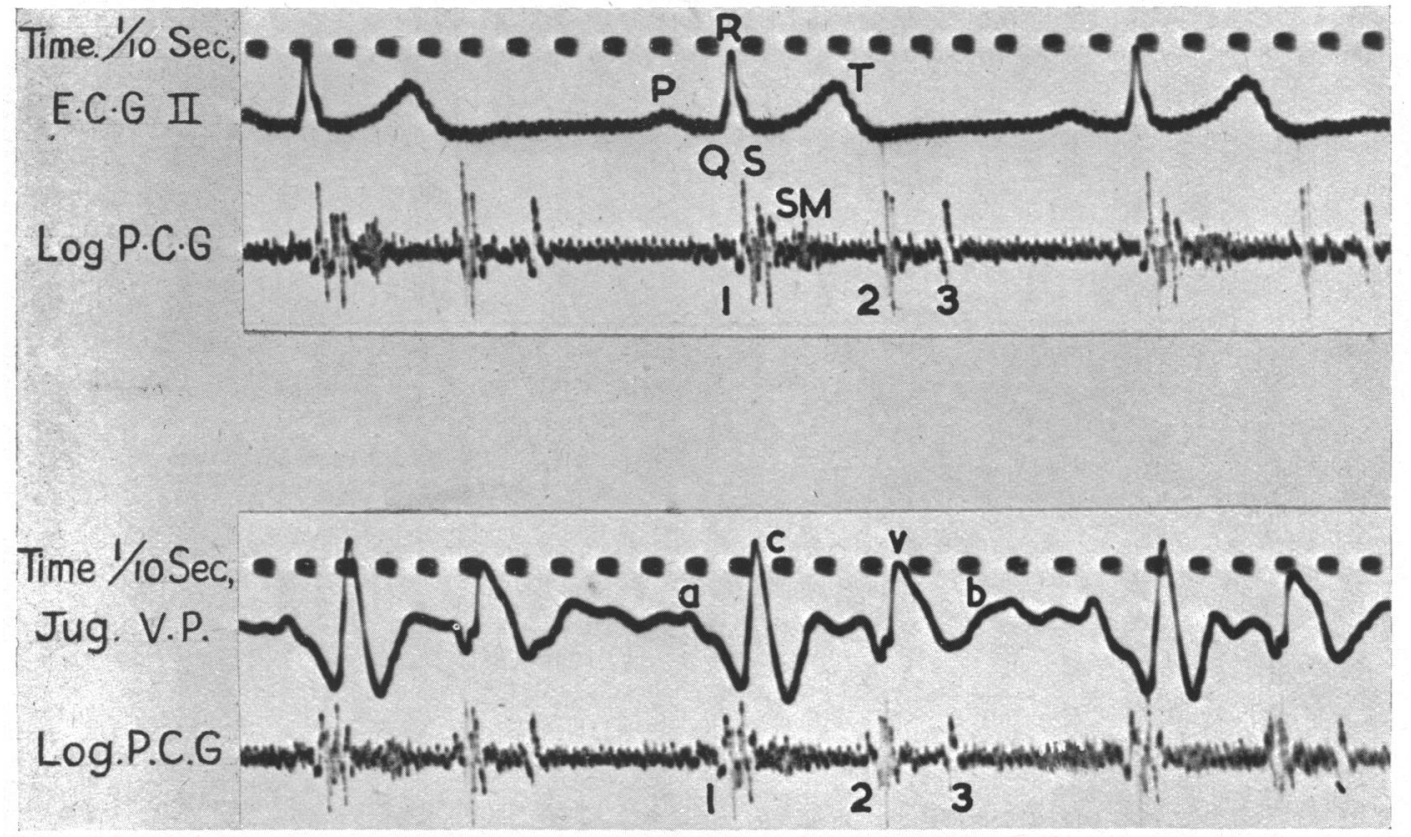

FIG. 4.-Logarithmic phonocardiograms from the mitral area of a healthy subject. Reference tracings: electrocardiogram, lead II (E.C.G. II) and jugular venous pulse (Jug. V.P.). 1, 2, 3, heart sounds; SM, systolic murmer.

This amplifier may be used either for detecting cardiographic potentials or for recording the jugular phlebogram. Cardiogram leads are connected directly to the input of the amplifier. The phlebogram may be recorded by placing over the external jugular vein a flat cup from a Mackenzie polygraph and connecting it by rubber tubing to a crystal microphone: the microphone in turn is connected to the input of the low frequency amplifier.

Cathode-ray Oscillograph and Camera. The cathode-ray oscillograph unit consists of a Cossor double-beam tube (type $89 \mathrm{~J})$ with power supply $(2000 \mathrm{v}$.) and voltage adjustments for brilliance and focus. A slow time base is provided to aid visual observation. This time base is stopped during recording while the recording paper or film is run past horizontally. 
Records are made with a Cossor camera (type 1428), with motor drive attachment, at a film speed of 1.2 inches a second on Ilford recording paper (B.P.1).

A small filament lamp is mounted near the oscillograph screen. A small synchronous motor and commutator activate the lamp 10 times a second to give timing marks.

\section{RECORDS}

Fig. 4 is an example of logarithmic phonocardiograms obtained with this instrument. These records were taken from a healthy subject with a well marked physiological third heart sound and an "innocent" systolic murmer.

\section{SUMMARY}

An adaptable apparatus is described for general-purpose phonocardiography. The instrument employs crystal microphones, electronic amplification, and cathode-ray tube recording. Either the venous pulse or the electrocardiogram is recorded simultaneously with the heart sounds.

Our thanks are due to Mr. I. Anderson who constructed the instrument, and to the Rankin Medical Research Fund of the University of Glasgow for defraying the cost of the apparatus. Fig. 1 is reproduced with the kind permission of the Editor of Glasgow Medical Journal.

\section{REFERENCES}

Boone, B. R. (1940). J. Lab. clin. Med., 25, 188

Cowen, E. D. H., and Parnum, D. H. (1949). Brit. Heart J., 11, 356.

Donovan, G. E. (1944). Lancet, 1, 500.

(1948). Lancet, 2, 401.

Fletcher, H. (1948). Speech and Hearing. 7th ed.; D. Van Nostrand Company, Inc., New York.

Kountz, W. B., Gilson, A. S., and Smith, J. R. (1940). Amer. Heart J., 20, 667.

Smith, J. R. Gilson, A. S., and Kountz, W. B. (1941). Amer. Heart J., 21, 17.

Williamson, D. T. N. (1951). The Williamson Amplifier. Iliffe and Sons, Ltd., London. 